\title{
Managed Care und Invalidenversicherung in der Wintersession
}

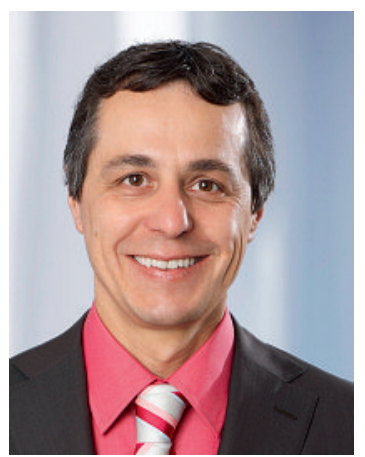

In der Wintersession hat das Parlament zwei für die Gesundheitspolitik wichtige Themen behandelt: im Ständerat (SR) die «integrierte Versorgung» und im Nationalrat (NR) die IV-Revision.

Bezüglich des KVG hat der SR im wesentlichen die Entscheide des NR vom Juni 2010 bestätigt. Die wichtigen Punkte wurden beibehalten: die Verbesserung des Risikoausgleichs, die differenzierte Kostenbeteiligung, der maximale jährliche Selbstbehalt (500 Franken innerhalb der Netze und 1000 Franken ausserhalb) sowie die Definition der «integrierten Versorgungsnetze». Ein solches Netz ist eine Organisation von Leistungserbringern, die sich zum Zweck einer Koordination der medizinischen Versorgung zusammenschliesst und die Patienten über die ganze Behandlungskette hinweg steuert. Eine solche Organisation schliesst einen Vertrag mit den Versicherern, muss das Qualitätsmanagement verdeutlichen und hat eine Budgetmitverantwortung. Der Bundesrat hat die Möglichkeit (aber nicht die Verpflichtung, wie es der NR wollte), Anforderungen an die notwendige Qualität der Versorgung festzulegen. Der SR hat die Verpflichtung der Versorgungsnetze zur Unabhängigkeit (z. B. von den Versicherern) gestri-

\section{Der Ständerat hat die Verbesserung der inte-}

grierten Versorgung unterstützt und die wesent-

\section{lichen Punkte des Nationalrates übernommen.}

\section{Vor der Schlussabstimmung sind nun noch die letzten Differenzen zu bereinigen.}

chen, obwohl dies von den Hausärzten mit Nachdruck gefordert worden war. Hinsichtlich der Forderungen der FMH hat der SR den Entwurf verbessert, indem die formelle Verpflichtung der Versicherer zum Angebot von Versicherungen für integrierte Versorgungsnetze gestrichen wurde und die Kostenbeteiligung von 20 auf $15 \%$ ausserhalb und von 10 auf $5 \%$ innerhalb der Netze reduziert wurde. Die Verpflichtung zur Budgetmitverantwortung wurde dagegen beibehalten. Im März wird der NR über eine neue Version befinden. Wenn das Gesetz verabschiedet wird, haben die Gegner die Möglichkeit, ein Referendum zu lancieren.
Der erste Teil (6a) der 6. Revision des Bundesgesetzes über die Invalidenversicherung wurde vom NR am 16. Dezember mit 116 gegen 63 Stimmen gutgeheissen. Da sich die finanzielle Lage der IV seit 1990 ständig verschlechtert hat und sie nun Schulden von 14 Milliarden aufweist, hat der Bundesrat ein dreistufiges Vorgehen festgelegt. Erstens wurde das jährliche Defizit mit der 4. und 5. Revision stabilisiert: Die Zahl der Neurenten wurde um 40\% reduziert. Zweitens läuft seit dem 1. Januar 2011 für sieben Jahre die Zusatzfinanzierung durch die MwSt.-Erhöhung, und es wurde ein separater IV-Fonds geschaffen, um die AHV-Rechnung von den IV-Schulden zu trennen. Mit diesen beiden Massnahmen können die Defizite und das Anwachsen der Schulden gestoppt werden, jedoch wird die IV dadurch nicht nachhaltig ins Gleichgewicht gebracht. Ohne das dritte Paket (Revision 6a und 6b) würde die IV ab 2018 erneut jährliche Defizite von 1,1 Milliarden aufweisen, und ihr Weiterbestehen für künftige Generationen wäre nicht gewährleistet.

Der erste Teil der 6. Revision umfasst vier Punkte: Die ersten beiden (eingliederungsorientierte Rentenrevision und Preissenkung im Bereich der Hilfsmittel) ermöglichen wesentliche Einsparungen, der dritte (neuer Finanzierungsmechanismus) entspricht hauptsächlich einer Einsparung für die IV dank zusätzlichen Bundesausgaben, während der vierte (Assistenzbeitrag) darauf ausgerichtet ist, Menschen mit einer Behinderung ein selbstständiges Leben zu ermöglichen.

Es ist eine Reduktion der Anzahl Renten um 5\% geplant: 17000 Personen werden bei der Suche nach einer geeigneten Stelle unterstützt. Da die Wirksamkeit von Quotensystemen nicht erwiesen ist, hat der NR Anreize - nicht aber eine Verpflichtung - für die Arbeitgeber eingeführt. Im weiteren hat er mit 111 gegen 69 Stimmen - entgegen der Auffassung der FMH - beschlossen, dass die Renten, die vor 2008 wegen unklaren psychischen Störungen (z. B. somatoforme Schmerzstörungen oder Fibromyalgie) zugesprochen wurden, in den nächsten drei Jahren prioritär geprüft werden müssen. Seit der 5. Revision berechtigen solche Diagnosen in der Regel nicht mehr zum Bezug einer Neurente, doch es musste eine Massnahme für die älteren Renten eingeführt werden (mit Ausnahme der Rentenbezüger von über 55 Jahren und denjenigen, die seit $<15$ Jahren eine Rente haben). Einmal mehr war das bestmögliche Gleichgewicht zwischen den Leistungsbezügern und den zahlenden Personen zu finden, zwischen den Gebern und den Nehmern. Eine grosse politische Herausforderung!

Dr. med. Ignazio Cassis Vizepräsident der FMH und Nationalrat 